\title{
Sixty Years of Stable Models
}

\author{
David Pearce \\ Universidad Rey Juan Carlos, Madrid, Spain
}

\section{Overview}

Twenty years ago Michael Gelfond and Vladimir Lifschitz published their celebrated paper on the stable model semantics of logic programs. Today, having built on and enlarged those key ideas of twenty years ago, answer set programming (ASP) has emerged as a flourishing paradigm of declarative programming, rich in theoretical advances and maturing applications. This is one aspect of the legacy of stable models, and a very important one. Another aspect, equally important, but somewhat farther from the limelight today, resides in the ability of stable models to provide us with a valuable method of reasoning - to give it a name let us call it stable reasoning. In the full version of this essay I examine some of the foundational concepts underlying the approach of stable models. I try to answer the question: "What is a stable model?" by searching for a purely logical grasp of the stability concept. In so doing, I shall discuss some concepts and results in logic from around 60 years ago. In particular, I look at questions such as:

- How does a notion of stability presented in a work on intuitionistic mathematics in 1947 relate to the Gelfond-Lifschtiz concept of 1988 ?

- How does the notion of constructible falsity published in 1949 help to explain certain properties of negation arising in the language of ASP?

- Why is a seminal paper by McKinsey and Tarski, published in 1948, important for understanding the relations between answer sets and epistemic logic?

Relating stable models and answer sets to logical concepts and results from the 1940s and even earlier sets the stage for a second line of discussion. I shall consider different techniques for studying the mathematical foundations of stable reasoning and ASP. One of these is based on classical, propositional and predicate logic. Its main advantage is its familiarity, its wealth of results and its suitability for rapid prototyping. Its drawback is that it lies, in a sense that can be made precise, one level removed from the action. We first have to translate, manipulate and modify, before we obtain relevant representations in classical logic that we could have obtained in simpler fashion using a non-classical logic. The second approach is based directly on a well-known non-classical logic. I use it to make some recommendations not only how stable reasoning and the foundations of ASP can best be grasped and further studied, but also on some specific topics for future research. 